\title{
Stable single-layer structure of group-V elements
}

\author{
F. Ersan, ${ }^{1}$ E. Aktürk, ${ }^{1,2,3,{ }^{*}}$ and S. Ciraci ${ }^{3, \dagger}$ \\ ${ }^{1}$ Department of Physics, Adnan Menderes University, 09100 Aydin, Turkey \\ ${ }^{2}$ Nanotechnology Application and Research Center, Adnan Menderes University, 09010 Aydin, Turkey \\ ${ }^{3}$ Department of Physics, Bilkent University, 06800 Ankara, Turkey
}

(Received 5 September 2016; revised manuscript received 3 November 2016; published 14 December 2016)

\begin{abstract}
In addition to stable single-layer buckled honeycomb and washboard structures of group-V elements (or pnictogens $\mathrm{P}, \mathrm{As}, \mathrm{Sb}$, and $\mathrm{Bi}$ ) we show that these elements can also form two-dimensional, single-layer structures consisting of buckled square and octagon rings. An extensive analysis comprising the calculation of mechanical properties, vibration frequencies, and finite-temperature $a b$ initio molecular dynamics confirms that these structures are dynamically and thermally stable and suitable for applications at room temperature and above. All these structures are semiconductors with a fundamental band gap, which is wide for P but decreases with increasing row number. The effect of the spin-orbit coupling decreases the band gap and is found to be crucial for $\mathrm{Sb}$ and $\mathrm{Bi}$. These results are obtained from first-principles calculations based on density functional theory.
\end{abstract}

DOI: 10.1103/PhysRevB.94.245417

\section{INTRODUCTION}

The element(s) and its atomic structure are crucial for the physical and chemical properties of two-dimensional (2D) materials. Hopes to find a contender for graphene [1,2] have initiated searches for new 2D materials of other elements in diverse structures. The synthesis of a single layer of hexagonal h-BN [3] and h- $\mathrm{MoS}_{2}$ [4] has been achieved quickly since their parent layered crystals have weak interlayer interactions like graphite. In the meantime, the stability of similar single-layer (SL) hexagonal (h), as well as trigonal (T), structures of several transition-metal oxides and dichalcogenides has been predicted [5], showing the diversity of electronic and magnetic properties. On the other hand, the tremendous experience accumulated with silicon technology rendered the synthesis of SL hexagonal Si a priority research field even if this element has no three-dimensional (3D) layered allotrope like graphite. Interestingly, theoretical studies [6,7] have shown that $\mathrm{Si}$ and Ge can, in fact, form stable SL honeycomb structures with hexagonal lattices if alternating atoms at the corners are buckled to ensure the stability in two dimensions. Later, silicene and germanene were synthesized on $\mathrm{Ag}$ and $\mathrm{Au}$ substrates [8,9]. Theoretical studies went further to include compounds of group-IV elements, as well as compounds of III-V and II-VI elements, in the study of stability; the majority of them were shown to form stable SL honeycomb structures [10-13]. Other manifolds of zinc-blende compounds, such as tetragonal and $\mathrm{V}$ shaped, were also predicted [14]. More recently, AlN and GaN in honeycomb structures have also been synthesized $[15,16]$.

Apart from the honeycomb structures of graphene, other allotropes, such as graphynes and graphdiynes, were also considered [17-21]. Theoretical studies predicted that $\alpha$-, $\beta$-, and $\gamma$-graphynes are stable; while $\alpha$ - and $\beta$-graphynes preserve Dirac cones, $\gamma$-graphyne is a semiconductor due to the Kekule distortion effect [22,23]. Ram et al.[24] predicted a $s p^{2}$ hybridized 2D allotrope of carbon, named pentahexoctite,

\footnotetext{
*ethem.akturk@adu.edu.tr

†ciraci@fen.bilkent.edu.tr
}

with mechanical strength comparable to that of graphene. In addition, Zhang et al. [25] predicted five atomically thin, free-standing germanium selenide ( $\mathrm{GeSe}$ ) polymorphs, which have high kinetic stability and energy band gaps in the visible region. It has been shown that $\mathrm{MoS}_{2}$, which is normally a semiconductor in the SL honeycomb structure, possesses both massless Dirac and heavy fermions when it is formed in a planar, SL square/octagon (s/o) structure with a 2D square lattice [26].

After the synthesis of very thin films of phosphorus [27] researchers started to seek similar structures in other group-V elements or pnictogens. Recent theoretical studies have predicted that nitrogen [28], phosphorus [29-31], arsenic [32-35], antimony [36-39], bismuth [40-43], and compounds of group$\mathrm{V}$ elements [44] can form stable freestanding SL, planar as well as buckled honeycomb (b) structures similar to that of silicene and germanene and also other manifolds, such as SL symmetric (w) and asymmetric (aw) washboard structures, among others. These SL phases are named, respectively, nitrogene, phosphorene, arsenene, antimonene, and bismuthene. It should be noted that phosphorus, arsenic, antimony, and bismuth have stable 3D quasilayered parent crystals, which corroborate efforts to synthesize SL or very thin films (or multilayers) of these elements. Among the possible nonhoneycomb structures of phosphorus, Guan et al. [30] and $\mathrm{Wu}$ et al. [31] proposed a s/o-type structure consisting of square and octagon rings. However, neither dynamical, thermal, nor other stability analysis of these structures was performed to show whether they are really stable, and no relevant structural parameters, such as buckling distance, short and long bonds, bond angles, pertinent mechanical properties, critical energetics, etc., were provided to characterize the s/o structure. Recently, Kou et al. [45] pointed out that the buckled s/o structure (which they named the tetragonal bilayer) of $\mathrm{Bi}$ is stable and exhibits topological insulator features.

Earlier, we studied SL pnictogen phases consisting of planar and buckled honeycomb and symmetric and asymmetric washboard structures, whereby their dynamical and thermal stability, mechanical, electronic, magnetic, and optical 
properties were revealed and also their functionalization with selected single atoms and molecules was investigated [21,34$36,43]$. In those studies crucial effects of the structures on physical properties were revealed. In this paper, we present a systematic study of pnictogens in SL freestanding, buckled s/o structures. Since our tests resulted in an instability of SL nitrogene in the s/o structure, our study comprises only P, As, $\mathrm{Sb}$, and $\mathrm{Bi}$ within the group- $\mathrm{V}$ elements. Having ensured that they are dynamically and thermally stable, we calculated their mechanical and electronic properties using density functional theory (DFT) and compared them with the properties of other SL phases of pnictogens. These structures are semiconductors with wide to narrow band gaps, depending on the type of constituent group- $\mathrm{V}$ elements. The effect of the spin-orbit coupling becomes crucial for $\mathrm{s} / \mathrm{o}-\mathrm{Sb}$ and $\mathrm{s} / \mathrm{o}-\mathrm{Bi}$, but band energies calculated within DFT require correction. Interesting correlations of the physical properties of s/o structures among pnictogens are also revealed.

\section{METHOD}

Our theoretical analysis and predictions are obtained with first-principles pseudopotential calculations based on both the spin-polarized and spin-unpolarized density functional theory within the generalized gradient approximation (GGA) including van der Waals corrections [46]. Here we used projector augmented-wave (PAW) potentials [47], and the exchange-correlation potential is approximated with the Perdew-Burke-Ernzerhof (PBE) functional [48]. Numerical calculations were carried out using the VASP software [49]. A plane-wave basis set with kinetic energy cutoff is taken to be $\hbar^{2}|\mathbf{k}+\mathbf{G}|^{2} / 2 m=400 \mathrm{eV}$. The Brillouin zones (BZs) were sampled in the $\mathbf{k}$ space within the Monkhorst-Pack [50] scheme using a $16 \times 16 \times 1$ mesh. Atomic positions were optimized using the conjugate gradient method, where the total energy and atomic forces were minimized. The energy convergence value between two consecutive steps was chosen to be $10^{-5} \mathrm{eV}$. A maximum force of $0.002 \mathrm{eV} / \AA$ was allowed on each atom. The Gaussian-type Fermi-level smearing method is used with a smearing width of $0.01 \mathrm{eV}$. In the periodically repeating slab model, the vacuum spacing between the layers is set to $15 \AA$ to minimize the interlayer coupling. Phonon dispersion curves are obtained using the finite-displacement method (for $2 \times 2$ supercells) as implemented in the PHONOPY [51] code without spin-orbit coupling (SOC). Additionally, the thermal stability analysis of the optimized structures was tested using finitetemperature $a b$ initio molecular dynamics (MD) calculations. A Nosé thermostat was used, and Newton's equation of motion was integrated through the Verlet algorithm with time steps of $2 \mathrm{fs}$.

Self-consistent field calculations of the electronic energy bands and total and orbital projected densities of states corresponding to the optimized structure were carried out with and without SOC. Since the fundamental band gaps are underestimated by standard DFT, we applied a correction to the PBE results using the screened hybrid functional HSE06 method [52-55]. The screening length of HSE06 is taken as $\lambda=0.2 \AA^{-1}$, and the mixing rate of the Hartree-Fock exchange potential is set to 0.25 .

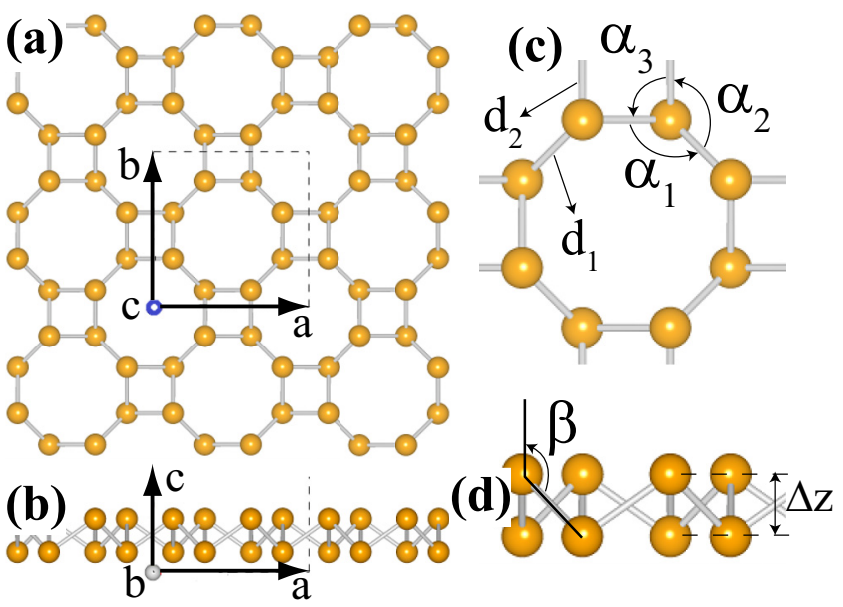

FIG. 1. Description of the atomic configuration and structural parameters of buckled s/o structure. (a) Top view of the atomic structure consisting of square and octagon rings. The square unit cell with lattice constants $\mathbf{a}=\mathbf{b}$ is delineated with dashed lines. (b) Side view consisting of two parallel atomic planes as a result of buckling. (c) An octagon ring of the s/o structure with relevant bonds $d_{1}$ and $d_{2}$ and bond angles $\alpha_{1}, \alpha_{2}$, and $\alpha_{3}$. (d) Side view of the octagon showing the buckling parameter $\Delta z$ and bond angle $\beta$.

\section{STRUCTURE AND ENERGETICS}

In Fig. $1 \mathrm{SL}$ the buckled s/o structure together with the major structural parameters and rectangular unit cell is shown. Even if the $x y$ projection of the structure appears to be formed from square and octagon rings, it actually consists of buckled square and octagon rings. The stability of the structure is maintained by the buckling of the planar structure, whereby atoms at alternating corners are buckled by $\Delta z$. In this way, a SL structure consists of two atomic planes. All corners of the rings are occupied by the same atoms of group- $\mathrm{V}$ elements, namely, $\mathrm{P}, \mathrm{As}, \mathrm{Sb}$, and $\mathrm{Bi}$. The unit cell comprises eight atoms at the corners of an octagon, which are connected by bonds, four of them, $d_{1}$, are shared by four adjacent octagons. The remaining four, $d_{2}$, are shared by square rings. In this way one distinguishes two different bonds, $d_{1}$ and $d_{2}$, where $d_{1}$ is slightly shorter than $d_{2}$. The percentage difference, $d_{2}-d_{1} / d_{1}$, increases from $0.9 \%$ to $3 \%$ as one goes from $\mathrm{P}$ to $\mathrm{Bi}$. The average of $d_{1}$ and $d_{2}$ of s/o- $X$ approximately equals the bond length $d$ of $\mathrm{b}-X$. The $\mathrm{s} / \mathrm{o}-X$ is an open structure relative to $\mathrm{b}-X$ with the ratio of the areal atom density being 0.8 for $\mathrm{P}$. This ratio decreases with increasing the row number of $X$ and becomes 0.75 for $\mathrm{Bi}$. A similar analysis can be carried out also using the areal packing fraction. All atoms are threefold coordinated in the s/o- $X$ structure; the bonds connecting any atom to its three nearest neighbors are not coplanar. Consequently, the hybridization of bonds deviates from $s p^{2}$ and acquires also a $p_{z}$ orbital contribution.

Structural parameters and lattice constants presented in Fig. 1 are optimized. The energetics, elastic constants, phonon frequencies, and electronic structures are calculated in terms of these optimized lattice constants. In Table I the values of structural parameters and elastic constants calculated for $\mathrm{s} / \mathrm{o}-X$ structures are compared with those of $\mathrm{b}-X, \mathrm{w}-X$, and 
TABLE I. Comparison of the calculated values of s/o- $X$ structures with those of $\mathrm{b}-X, \mathrm{w}-X$, and aw- $X(X=\mathrm{P}, \mathrm{As}, \mathrm{Sb}$, and $\mathrm{Bi})$ structures. Values without references are calculated in the present study. Entries are square-lattice constants $a=b$, bond lengths $d_{i}(i=1,2)$, selected bond angles $\alpha_{i}(i=1,2,3)$ and $\beta$, buckling parameter $\Delta z$, in-plane stiffness $C_{x}, C_{y}$; Poisson's ratios $v_{x y}, v_{y x}$.

\begin{tabular}{|c|c|c|c|c|c|c|c|}
\hline & Structure & $\begin{array}{l}\text { Lattice } \\
(\AA)\end{array}$ & $\begin{array}{l}d_{i} \\
(\AA)\end{array}$ & $\begin{array}{l}\alpha_{i}, \beta \\
(\mathrm{deg})\end{array}$ & $\begin{array}{l}\Delta z \\
(\AA)\end{array}$ & $\begin{array}{c}C_{x}=C_{y} \\
\left(\mathrm{~J} / \mathrm{m}^{2}\right)\end{array}$ & $v_{x y}=v_{y x}$ \\
\hline \multirow[t]{4}{*}{$\mathrm{P}$} & $\mathrm{s} / \mathrm{o}$ & $a=b=6.54$ & $d_{1}=2.26, d_{2}=2.28$ & $\begin{array}{c}\alpha_{1}=100.6, \alpha_{2}=100.7 \\
\alpha_{3}=72.4, \beta=123.7\end{array}$ & 1.25 & $C_{x, y}=32.06$ & $v_{x y}=0.532$ \\
\hline & $\mathrm{b}$ & $a=b=3.28,3.28$ & $2.27,2.26[56]$ & $92.9,92.9[56]$ & $1.24,1.24[56]$ & $C_{x, y}=75.45$ & $v_{x y}=0.107$ \\
\hline & w & $a=3.31,3.30[57,58]$ & $d_{1}=2.22,2.22[57,58]$ & $96[57,58], 104[57,58]$ & $2.51[58]$ & $C_{x}=44[58], 41.3[59]$ & $v_{x y}=0.17[58]$ \\
\hline & & $b=4.55,4.62[57,58]$ & $d_{2}=2.25,2.26[57,58]$ & $96.1,103.5$ & 2.12 & $\mathrm{C}_{y}=166[58], 106.4[59]$ & $v_{y x}=0.62[58]$ \\
\hline \multirow[t]{4}{*}{ As } & $\mathrm{s} / \mathrm{o}$ & $a=b=7.06$ & $d_{1}=2.48, d_{2}=2.52$ & $\begin{array}{l}\alpha_{1}=99.1, \alpha_{2}=99.4 \\
\alpha_{3}=71.8, \beta=124.7\end{array}$ & 1.42 & $C_{x, y}=20.87$ & $v_{x y}=0.599$ \\
\hline & $\mathrm{b}$ & $a=b=3.60,3.61[32]$ & $2.50,2.50[32]$ & $91.9,92.2[32]$ & 1.40 & $C_{x, y}=51.41$ & $v_{x y}=0.165$ \\
\hline & w & $a=3.67,3.68[32]$ & $d_{1}=2.50,2.50[32]$ & $94.6[32], 100.8$ [32] & & $\mathrm{C}_{x}=29[33]$ & \\
\hline & & $b=4.72,4.77[32]$ & $d_{2}=2.47,2.49[32]$ & $94.7,100.6$ & 2.38 & $C_{y}=74.7[33]$ & \\
\hline \multirow[t]{4}{*}{$\mathrm{Sb}$} & $\mathrm{s} / \mathrm{o}$ & $a=b=8.01$ & $d_{1}=2.85, d_{2}=2.91$ & $\begin{array}{c}\alpha_{1}=97.4, \alpha_{2}=97.5 \\
\alpha_{3}=70.6, \beta=126.1\end{array}$ & 1.67 & $C_{x, y}=11.55$ & $v_{x y}=0.663$ \\
\hline & $\mathrm{b}$ & $a=b=4.04,4.12[60]$ & $2.87,2.89[60]$ & $89.6,89[36]$ & $1.67,1.65[60]$ & $C_{x, y}=34.56$ & $v_{x y}=0.192$ \\
\hline & aw & $a=4.78,4.74[39]$ & $d_{1}=2.85,2.87$ [39] & 95.3 [39], 102.4 [39] & & $C_{x}=12[36]$ & $v_{x y}=0.36[36]$ \\
\hline & & $b=4.27,4.36[39]$ & $d_{2}=2.91,2.94[39]$ & $94.4,103.5$ & $0.38,2.82$ & $C_{y}=29[36]$ & $v_{y x}=1.20[36]$ \\
\hline \multirow[t]{4}{*}{$\mathrm{Bi}$} & $\mathrm{s} / \mathrm{o}$ & $a=b=8.40$ & $d_{1}=2.99, d_{2}=3.08$ & $\begin{array}{l}\alpha_{1}=96.6, \alpha_{2}=96.7 \\
\alpha_{3}=70.4, \beta=126.7\end{array}$ & 1.78 & $C_{x, y}=7.61$ & $v_{x y}=0.728$ \\
\hline & $\mathrm{b}$ & $a=b=4.19,4.33[60]$ & $3.01,3.09[60]$ & 87.11 & $1.80,1.71[60]$ & $C_{x, y}=29.61$ & $v_{x y}=0.232$ \\
\hline & aw & $a=4.87,4.94[43]$ & $d_{1}=3.08,3.11[43]$ & $92.0,107.4$ & $0.5[43]$ & $C_{x}=10.03[43]$ & $v_{x y}=0.261[43]$ \\
\hline & & $b=4.44,4.55[43]$ & $d_{2}=3.03,3.10[43]$ & $92.2,106.7$ & $0.57,2.72$ & $C_{y}=25.5[43]$ & $v_{y x}=0.648[43]$ \\
\hline
\end{tabular}

aw- $X(X=\mathrm{P}, \mathrm{As}, \mathrm{Sb}$, and $\mathrm{Bi})$ structures. Notably, the lattice constants $a=b$, bond lengths $d_{1,2}$, and the buckling $\Delta z$ increase with increasing the row number of the $X$ atom. The average bond lengths of $\mathrm{s} / \mathrm{o}-X, \mathrm{~b}-X$, and $\mathrm{w}-X$ structures are approximately equal, but $\mathrm{s} / \mathrm{o}-X$ and $\mathrm{b}-X$ have a relatively smaller buckling parameter. In the rest of the paper, the identification "SL buckled" in front of s/o will be dropped for the sake of brevity.

In Fig. 2 the isosurfaces of the total charge densities of s/o-As, b-As, and w-As are shown. Because of differences in the hybridization of hybrid orbitals in different bonds in s/oAs, the overall charge-density distribution of these structures appears to be different.

As for the energetics of these structures, the cohesive energy and the formation energy at $T=0$ are relevant. The cohesive energy per atom of $\mathrm{s} / \mathrm{o}-X$ structures, $E_{c}=$ $E_{T}[X]-E_{T}[s / o-X] / 8$, is obtained from the difference between the total energy of a free $X$ atom and that of the corresponding s/o- $X$ per atom. By definition $E_{c}>0$ indicates binding. The formation energy $E_{f}$ at $T=0$ is obtained by
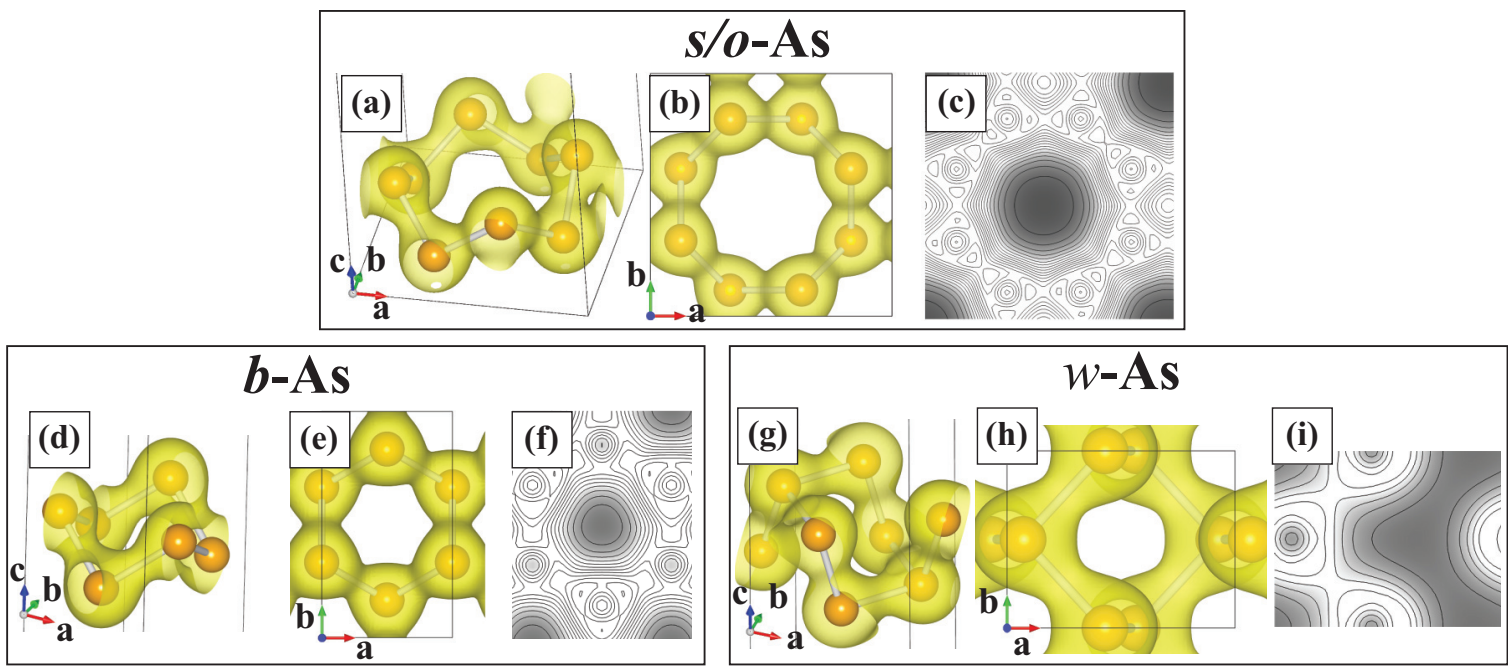

FIG. 2. Total charge-density plots of buckled s/o-As, b-As, and w-As. (a) Tilted view for s/o-As. (b) Top view of the charge-density isosurfaces on an octagon ring. (c) Contour plots of the total charge density on a plane passing through the $x y$ plane (parallel to atomic planes), which bisects $\Delta z$. (d)-(f) The same for b-As and (g)-(i) the same for w-As. Isosurface and contour spacing values are 0.05 and 0.015 electrons $/ \AA^{3}$, respectively. 
TABLE II. Comparison of the calculated cohesive energies $E_{c}$ of s/o- $X$ structures with the cohesive energies of $3 \mathrm{D}$ bulk crystals, b- $X$, $\mathrm{w}-X$, and aw- $X(X=\mathrm{P}, \mathrm{As}, \mathrm{Sb}$, and $\mathrm{Bi})$ structures. Formation energies at $T=0 \mathrm{~K}, E_{f}$, are given in parentheses.

\begin{tabular}{|c|c|c|c|c|c|}
\hline & Bulk (eV/atom) & b- $X$ (eV/atom) & $\mathrm{w}-X$ (eV/atom) & aw- $X$ (eV/atom) & s/o- $X$ (eV/atom) \\
\hline \multirow[t]{5}{*}{$\mathrm{P}$} & 3.67 & $3.55(-0.12)$ & $3.59(-0.08)$ & & $3.43(-0.24)$ \\
\hline & $3.33[61]$ & $3.47[62]$ & $3.48[57]$ & & \\
\hline & $3.49-4.74[63]$ & $3.42[64]$ & & & \\
\hline & $3.43[65]$ & $3.29[65]$ & $3.30[65]$ & & \\
\hline & $3.79[66]$ & & & & \\
\hline \multirow[t]{5}{*}{ As } & 3.30 & $3.14(-0.16)$ & $3.13(-0.17)$ & & $2.98(-0.32)$ \\
\hline & $2.99[32]$ & $2.99[32]$ & $2.95[32]$ & & \\
\hline & & 3.15 [67] & $3.13[67]$ & & \\
\hline & & $2.95[68]$ & & & \\
\hline & & 2.96 [69] & & & \\
\hline \multirow[t]{3}{*}{$\mathrm{Sb}$} & 3.13 & $2.86(-0.27)$ & & $2.89(-0.24)$ & $2.69(-0.44)$ \\
\hline & $2.75[70,71]$ & $4.26[39]$ & & $4.29[39]$ & \\
\hline & $3.12[36]$ & $2.87[36]$ & $2.88[36]$ & $2.89[36]$ & \\
\hline \multirow[t]{4}{*}{$\mathrm{Bi}$} & 3.12 & $2.77(-0.36)$ & & $2.84(-0.28)$ & $2.57(-0.56)$ \\
\hline & $3.22[41]$ & $1.95[43]$ & $1.97[43]$ & $1.97[43]$ & \\
\hline & & $1.97[40]$ & & & \\
\hline & & $4.45[45]$ & & & $4.25[45]$ \\
\hline
\end{tabular}
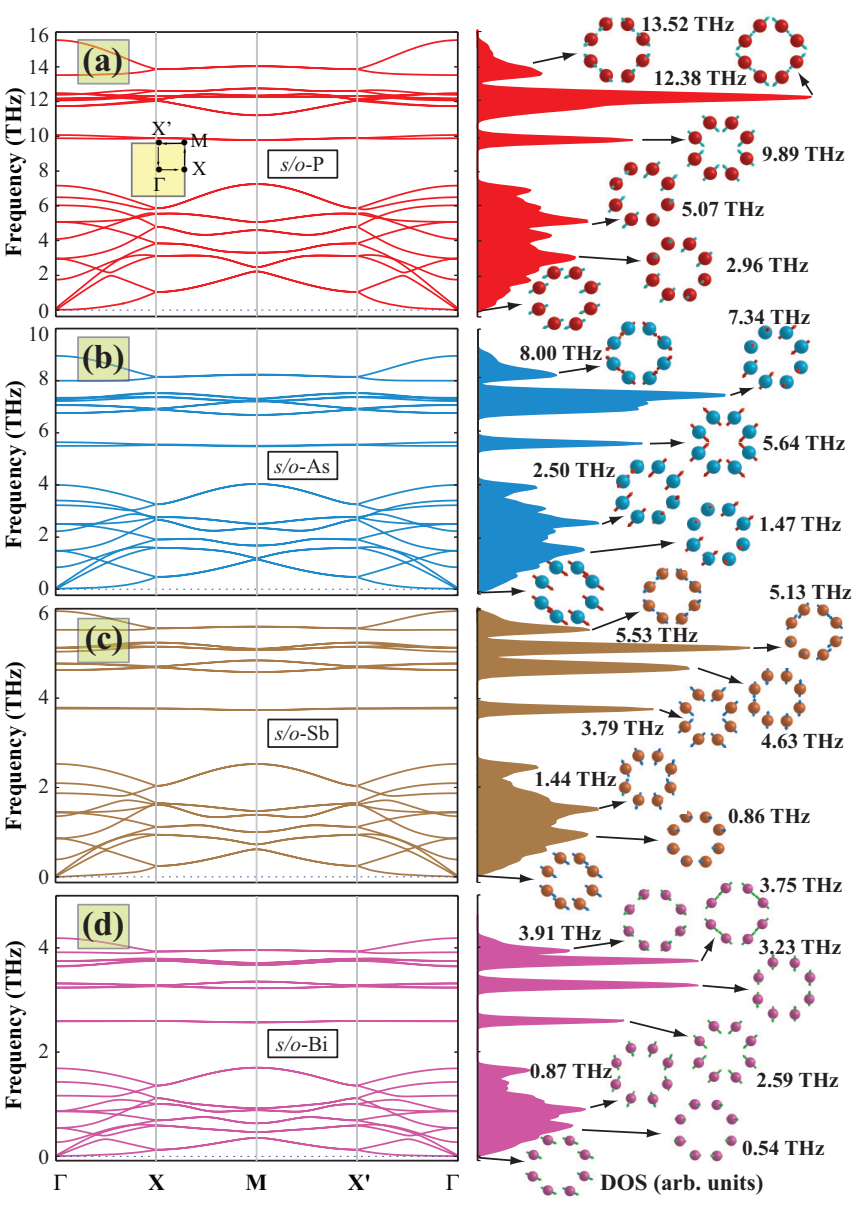

FIG. 3. (a)-(d) Phonon bands, i.e., frequency $f$ versus $\mathbf{k}$ along the selected symmetry axis of the square Brillouin zone shown by the inset and corresponding total densities of states. Special modes corresponding to selected singularities are shown in the insets. subtracting the cohesive energy of the 3D bulk crystal of the $X$ element from the cohesive energy of the s/o- $X$ structure. In Table II, the energetics of 3D bulk, s/o- $X, \mathrm{~b}-X, \mathrm{w}-X$, and aw- $X$ are compared. The cohesive energy of the s/o- $X$

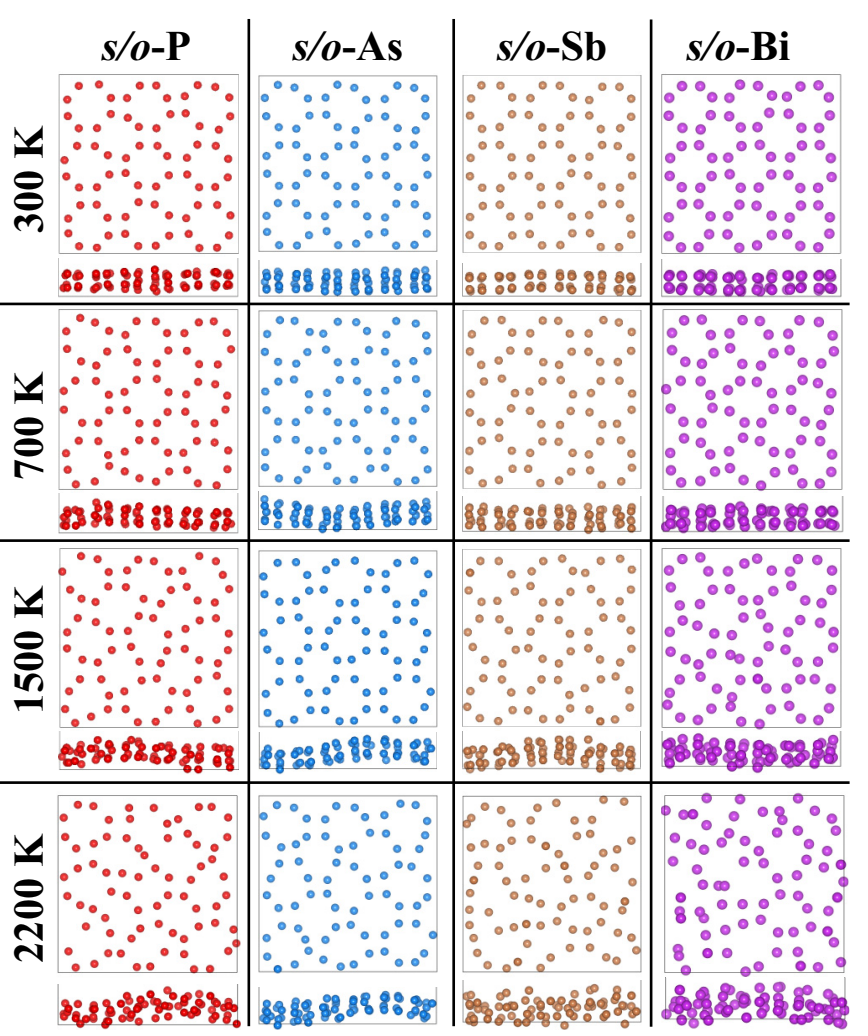

FIG. 4. Top and side views of the snapshots of the atomic configurations of s/o- $X(X=\mathrm{P}, \mathrm{As}, \mathrm{Sb}$, and $\mathrm{Bi})$ structures taken from MD simulations carried out at different temperatures. 

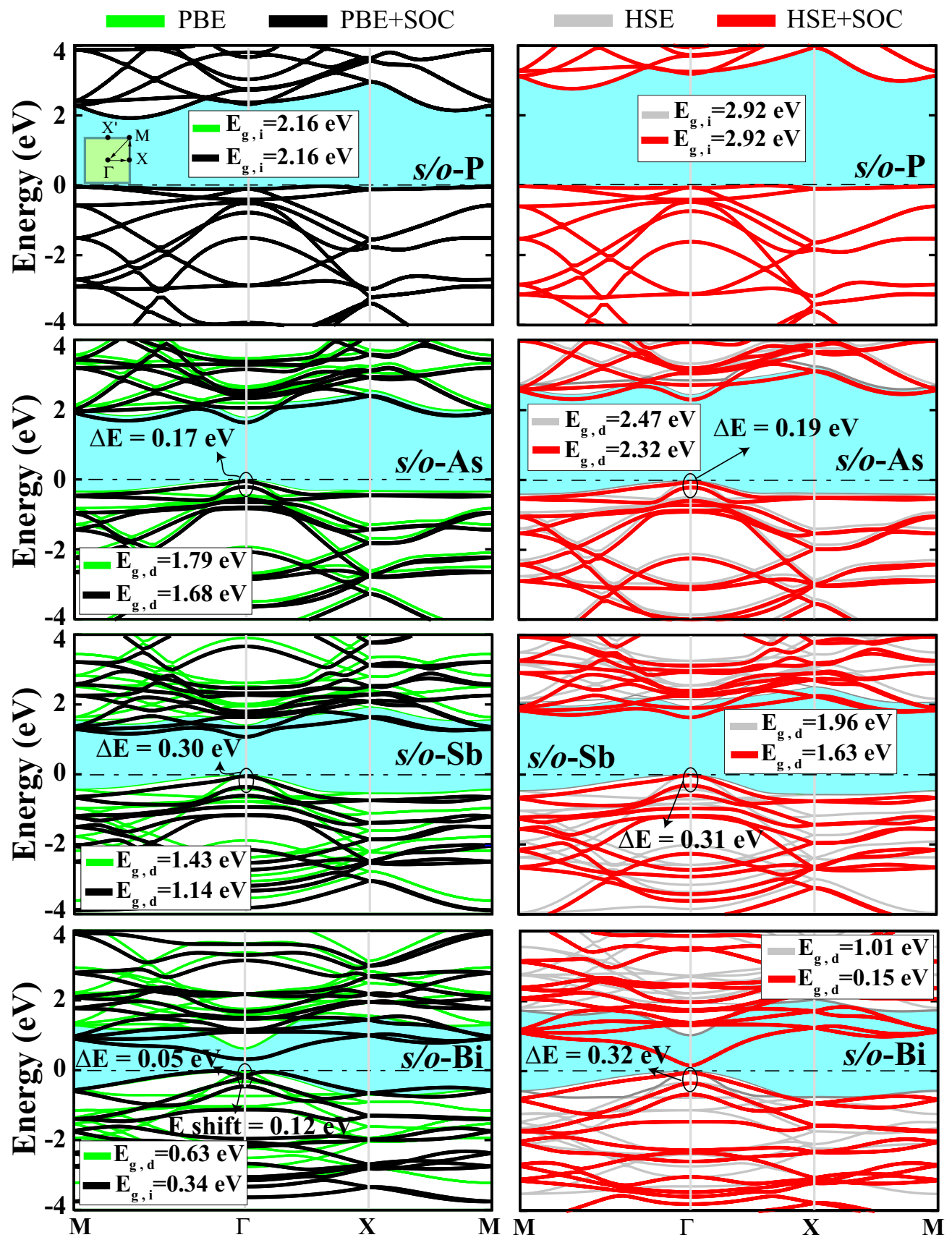

FIG. 5. Energy band structures of s/o- $X$ ( $X=\mathrm{P}, \mathrm{As}, \mathrm{Sb}$, and Bi) structures calculated with PBE (green), PBE+SOC (black), HSE (gray), and HSE+SOC (red). The zero of the energy is set at the top of the valence bands. The band gaps between conduction and valence bands are shaded, and fundamental band gaps are indicated as direct $E_{g, d}$ or indirect $E_{g, i} . \Delta E$ is the spin-orbit splitting at the top of the valence band. $E_{\text {shift }}$ is the energy difference of the state at the top of the valence band after Rashba splitting.

structure is consistently $0.16-0.27 \mathrm{eV}$ per atom smaller than the most energetic SL structure of $X$ elements. However, this small difference does not imply that s/o- $X$ structures cannot be synthesized and/or they cannot remain stable at room temperature. All these structures have positive binding energy but have negative formation energy. Accordingly, the optimized s/o- $X$ phases may correspond to local minima on the Born-Oppenheimer surfaces like other structures. This situation necessitates an extensive stability analysis of each $\mathrm{s} / \mathrm{o}-X$ structure in the next section.

\section{STABILITY ANALYSIS}

Our analysis of stability starts with the calculation of the mechanical properties of the s/o- $X$ optimized structures. To this end we calculate the in-plane stiffness [72] $C_{x, y}=$ $(1 / A) \partial E_{T}^{2} / \partial \epsilon_{x, y}^{2}$ and Poisson's ratio $v_{x y}=\epsilon_{y} / \epsilon_{x}$. In these expressions, $A$ is the area of the unit cell, $E_{T}$ is the total energy per cell of the s/o- $X$ structure, and $\epsilon_{x}$ is the uniaxial strain along the $x$ axis. We note that $C_{x}=C_{y}$ and $v_{x y}=v_{y x}$. Calculated values listed in Table I indicate that the in-plane 
stiffness of the s/o- $X$ structure is smaller than that of $\mathrm{b}-X$, $\mathrm{w}-X$, and aw- $X$ structures. This is mainly due to the fact that $\mathrm{s} / \mathrm{o}-X$ is more open than other structures. Nevertheless, all $\mathrm{s} / \mathrm{o}-X$ phases have positive in-plane stiffness, which decreases with increasing the row number of $X$. Note that the difference between in-plane stiffnesses of $\mathrm{b}-X$ and s/o- $X$ decreases with increasing the row number of $X$. This situation complies with the fact that the areal atom density also decreases as the row number of $X$ increases.

We will deduce from the present analysis whether s/o$X$ structures correspond to local minima in the BornOppenheimer surface and whether these minima are deep enough to provide stability against thermal excitations. Stability tests in the present study cover dynamical stability at $T=0 \mathrm{~K}$ and high-temperature thermal stability. In the former, the vibration frequencies $f(\mathbf{k})=\Omega(\mathbf{k}) / 2 \pi$ of a given $\mathrm{s} / \mathrm{o}-X$ structure in the $\mathbf{k}$ space are calculated. The structure is considered dynamically stable at $T=0 \mathrm{~K}$ only if all vibration frequencies in the $\mathrm{BZ} f(\mathbf{k})>0$. The calculated vibration frequencies of all s/o- $X(X=\mathrm{P}, \mathrm{As}, \mathrm{Sb}$, and $\mathrm{Bi})$ structures are found to be positive and hence confirm the dynamical stability of these four structures at $T=0$. In Fig. 3, we present the dispersions of the vibration frequencies $f=(\mathbf{k})$ along symmetry directions (or phonon bands) and the densities of states. The modes corresponding to specific frequencies are also described. The overall distributions of the vibration frequencies are similar in s/o- $X$ structures, except that their widths and the frequencies of singular points in the densities of states increase as the row numbers of elements decreases. It should be noted that for elements with low row numbers, the $X-X$ bonds are relatively shorter and, conversely, force constants are relatively stronger, leading to relatively higher vibration frequencies.

The thermal stability at high temperature, which is crucial from the technological application point of view of s/o- $X$ structures, indicates that local minimum of a s/o- $X$ structure is deep. This is tested by ab initio MD calculations. Here any s/o$X$ structure preserving its overall shape at high temperature for a time scale of picoseconds and keeping its optimized atomic configuration as $T \rightarrow 0$ can ensure that it is stable at room temperature and perhaps slightly above. In Fig. 4 we show the snapshots of atomic configurations of $\mathrm{s} / \mathrm{o}-X$ structures taken from MD simulations at various temperatures. In the course of MD iterations lasting 2 ps at $1500 \mathrm{~K}$ the identity of the s/o structure remains, especially for $\mathrm{P}$ and As. Severe and irreversible distortions start to occur from $1500 \mathrm{~K}$ on for heavier elements for $\mathrm{Sb}$ and $\mathrm{Bi}$.

Based on calculated values of mechanical properties and dynamical and thermal stability tests, which were performed on the picosecond time scale at temperatures as high as 2200 $\mathrm{K}$, one concludes that four freestanding $\mathrm{s} / \mathrm{o}-X$ structures considered in this paper can be stable at room temperature and at least slightly above it. This conclusion renders the electronic properties of these four s/o structures worthy of investigation for possible applications.

\section{ELECTRONIC PROPERTIES}

Understanding the physical properties, in particular the electronic energy bands of stable $\mathrm{s} / \mathrm{o}-X$ structures, is the prime motivation of the present study. The electronic structure of the optimized and stable s/o- $X(X=\mathrm{P}, \mathrm{As}, \mathrm{Sb}$, and $\mathrm{Bi})$ structures will be investigated in this section. The electronic band structures calculated along the selected directions of the BZ within PBE, PBE+SOC, HSE, and HSE+SOC are presented in Fig. 5. Comparing these bands, the effects of spin-orbit coupling and HSE corrections to DFT values are revealed. In addition, results of recent calculations on the energy bands of different structures, such as $\mathrm{b}-X, \mathrm{w}-X$, and aw- $X(X=\mathrm{P}, \mathrm{As}, \mathrm{Sb}$, and $\mathrm{Bi})$, will be compared in order to better understand the present s/o structure. Values of the fundamental band gaps calculated using the PBE method together with the band gaps of the $\mathrm{b}-X, \mathrm{w}-X$, and aw- $X$ structures calculated earlier are listed in Table III.

The fundamental band gap calculated using PBE is indirect and is $2.16 \mathrm{eV}$ for $\mathrm{s} / \mathrm{o}-\mathrm{P}$ but becomes direct for $\mathrm{s} / \mathrm{o}$ structures of heavier group- $\mathrm{V}$ elements and decreases as the row numbers increase. $\mathrm{s} / \mathrm{o}-\mathrm{Bi}$ has the smallest $\mathrm{PBE}$ band gap, $E_{g, d}=$ $0.63 \mathrm{eV}$. The effect of SOC is negligible for s/o-P but becomes significant starting with s/o-As and increases as the row number of group- $\mathrm{V}$ elements increases. Upon inclusion of SOC in PBE calculations, the fundamental band gaps decrease by $0.11,0.29$, and $0.29 \mathrm{eV}$ in s/o-As, s/o-Sb, and s/o-Bi, respectively. Moreover, through the Rashba-type splitting, the fundamental band gap of s/o-Bi changes from direct to indirect and becomes $E_{g, i}=0.34 \mathrm{eV}$. The present values of

TABLE III. Fundamental band gaps of s/o- $X(X=\mathrm{P}, \mathrm{As}, \mathrm{Sb}$, and Bi) calculated using PBE, PBE+SOC, HSE, and HSE+SOC. Available PBE values calculated for $\mathrm{b}-X, \mathrm{w}-$, and aw- $X$ are also given for the sake of comparison. Direct and indirect types of the band gap are indicated by (i) and (d), respectively.

\begin{tabular}{|c|c|c|c|c|c|}
\hline \multirow[b]{2}{*}{ Method } & \multirow[b]{2}{*}{ Structure } & \multicolumn{4}{|c|}{$E_{\text {gap }}(\mathrm{eV})$} \\
\hline & & $\mathrm{P}$ & As & $\mathrm{Sb}$ & $\mathrm{Bi}$ \\
\hline \multirow[t]{4}{*}{ PBE } & $\mathrm{s} / \mathrm{o}$ & 2.16 (i) & 1.79 (d) & $1.43(\mathrm{~d})$ & $0.63(\mathrm{~d})$ \\
\hline & $\mathrm{b}$ & 1.91 (i), 1.98 [65] & 1.57 (i), 1.64 [32] & 1.07 (i), 1.18 [73] & $0.49(\mathrm{~d}), 0.55[43]$ \\
\hline & $\mathrm{w}$ & 0.82 (d), $0.90[65]$ & 0.77 (i), 0.83 [32] & 0.37 (i), 0.54 [73] & $0.16(d), 0.16[43]$ \\
\hline & aw & & & 0.15 (i), $0.16[36]$ & $0.29(\mathrm{~d}), 0.31[43]$ \\
\hline $\mathrm{PBE}+\mathrm{SOC}$ & s/o & 2.16 (i) & $1.68(d)$ & $1.14(\mathrm{~d})$ & 0.34 (i) \\
\hline HSE & s/o & 2.92 (i) & $2.47(\mathrm{~d})$ & $1.96(d)$ & $1.01(\mathrm{~d})$ \\
\hline $\mathrm{HSE}+\mathrm{SOC}$ & s/o & 2.92 (i) & $2.32(\mathrm{~d})$ & $1.63(\mathrm{~d})$ & $0.15(\mathrm{~d})$ \\
\hline
\end{tabular}


the fundamental band gap of s/o-Bi are in good agreement with those reported by Kou et al. [45]

Since the fundamental band gaps are normally underestimated by DFT, the band structures are also calculated using the HSE correction. After HSE correction to the PBE bands, the fundamental band gaps of $\mathrm{s} / \mathrm{o}-X$ structures generally increase, but the direct/indirect character of the fundamental band gaps is unaltered. The effect of HSE correction is large for s/o-P and is $0.76 \mathrm{eV}$ but decreases with increasing the row number of $X$. Namely, the correction is $0.68,0.53$, and $0.38 \mathrm{eV}$ for s/o-As, s/o-Sb, and s/o-Bi, respectively. After the inclusion of SOC in the HSE correction, the band gap of s/o-P stays unaltered and remains indirect. However, even if its character is unchanged upon the inclusion of SOC, the band gaps decrease by $0.15,0.33$, and $0.86 \mathrm{eV}$ for s/o-As, s/o-Sb, and s/o-Bi, respectively. Accordingly, the direct fundamental band gap of $\mathrm{s} / \mathrm{o}-\mathrm{Bi}$ is predicted to be $0.15 \mathrm{eV}$, which may lead to crucial properties relevant to topological insulator character.

The total and orbital projected densities of states of s/o- $X$ structures are presented in Fig. 6 . A sharp peak just at the edge of the valence band of s/o-P is due to the flat bands of $p_{z}$ orbitals located at the top of the valence band. Contributions of $s, p_{x}$, and $p_{y}$ orbitals to the states at the edge of the valence band are relatively smaller. However, the contribution of the $p_{z}$ orbital to the states at the edge of the conduction band becomes comparable to those of the $p_{x}$ and $p_{y}$ orbitals. The distributions of the state density near the edge of the valence bands of s/o-As, s/o-Sb, and s/o-Bi differ from that of s/o-P due to the energy decrease of the flat $p_{z}$ band along $M-\Gamma-X$ symmetry directions of the BZ. Hence, contrary to s/o-P, the contributions of the $p_{x}$ and $p_{y}$ orbitals to the states just at the top of the valence bands near the $\Gamma$ point exceed that of the $p_{z}$ orbital for s/o-As and s/o-Sb. This is reversed for the edge of the conduction band, where the contribution of the $p_{z}$ orbital is relatively larger. Moreover, this situation is unaltered with the inclusion of SOC in the PBE calculations. Notably, for PBE bands the contributions of the $p_{x}, p_{y}$, and $p_{z}$ orbitals to the band edges of s/o-Bi are reminiscent of those of s/o-As and $\mathrm{s} / \mathrm{o}-\mathrm{Sb}$ discussed above. However, once SOC is included in the PBE bands of $\mathrm{s} / \mathrm{o}-\mathrm{Bi}$, the order of the $p_{z}$-orbital contribution to the band edges is inverted.

\section{DISCUSSION AND CONCLUSIONS}

While 2D SL structures of group-V elements in buckled honeycomb and washboard structures are gaining increasing research interest, here we predict another structure, which consists of buckled squares and octagon rings specified as $\mathrm{s} / \mathrm{o}-X$. This structure maintains group-V elements threefold coordinated like buckled honeycomb and washboard structures and has an even number of atoms in the primitive square lattice. Our analysis based on calculated mechanical properties, positive vibration frequencies, and high-temperature $a b$ initio molecular dynamic simulations indicates that suspended s/o$X$ structures are stable at room temperature and at least above it. Additionally, all of these structures are semiconductors with fundamental band gaps ranging from 3 to $0.15 \mathrm{eV}$. This makes s/o structures of group-V elements suitable for possible diverse applications in 2D electronics. In Fig. 7 we present the correlations of calculated values, the cohesive energy,
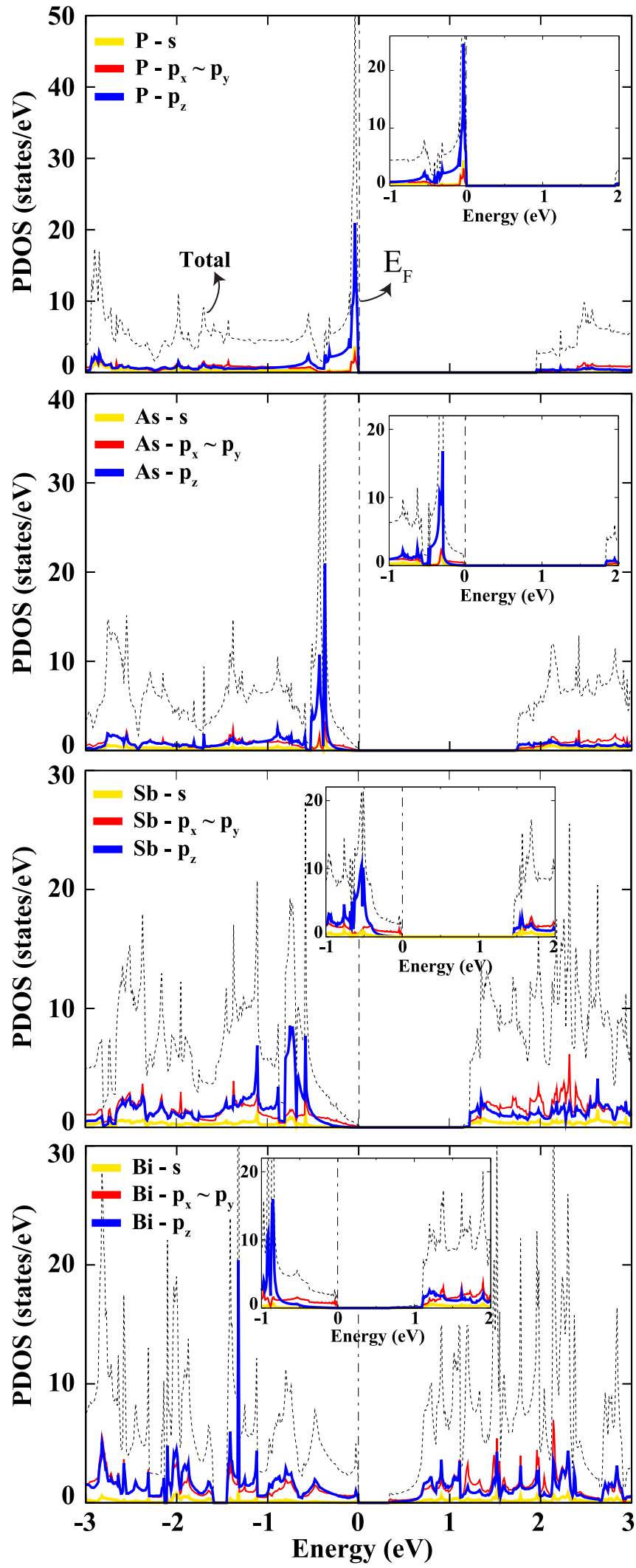

FIG. 6. Total and orbital projected densities of states of s/o- $X$ $(X=\mathrm{P}, \mathrm{As}, \mathrm{Sb}$, and $\mathrm{Bi})$ structures calculated using $\mathrm{PBE}$ and $\mathrm{PBE}+\mathrm{SOC}$. The zero of the energy is set at the top of the valence bands. The projected densities of states of s/o- $X$ structures calculated using PBE are shown by the inset near the edges of the fundamental band gap. 


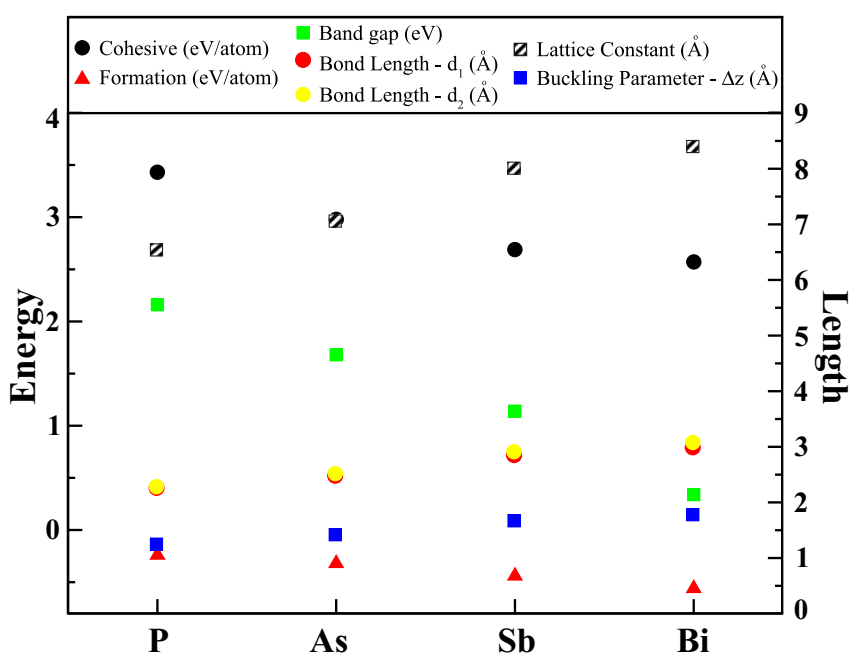

FIG. 7. Correlations among the calculated values of cohesive energy, formation energy, band gap and bond lengths of different $\mathrm{s} / \mathrm{o}-X$ structures treated in this study.

formation energy, fundamental band gap, bond lengths, lattice constants and bucklings parameters of s/o structures among group-V elements. While structural parameters, such as lattice constants, bond lengths and buckling parameters increase with increasing row number of the group- $\mathrm{V}$ elements, cohesive and formation energies, as well as fundamental band gap (PBE) decrease with increasing row number.

We found that the fundamental band gap of the s/o structure of any $X$ element calculated using PBE is always largest among those of other stable SL phases, b, w, and aw structures, of the same element. The effect of spin-orbit coupling on PBE bands is negligible for s/o-P but becomes significant for $\mathrm{s} / \mathrm{o}-\mathrm{Sb}$ and $\mathrm{s} / \mathrm{o}-\mathrm{Bi}$, where the band gap decreases by $\sim 0.3 \mathrm{eV}$. Upon HSE correction being applied to the PBE results, the band gap increases; the increment is as large as $\sim 0.8 \mathrm{eV}$ for $\mathrm{P}$ and As but is relatively smaller for $\mathrm{Sb}$ and $\mathrm{Bi}$. The band gaps normally decrease also with the inclusion of the spin-orbit coupling in the HSE correction. After the inclusion of the spin-orbit coupling in the HSE correction, the band gap of $\mathrm{s} / \mathrm{o}-\mathrm{Bi}$ decreases from 1.01 to $0.15 \mathrm{eV}$, and the dominant orbital character of the states at the top of the valence band switches from $p_{x}$ and $p_{y}$ to $p_{z}$. Strong spin-orbit coupling can induce features characteristic of a $2 \mathrm{D}$ topological insulator as in the asymmetric washboard structure of $\mathrm{Bi}$.

The overall picture of the charge-density plots of $\mathrm{s} / \mathrm{o}-X$ is reminiscent of that of $\mathrm{b}-X$. However, significant differences in the angles $\alpha_{i}, i=1-3$, and $\beta$ give rise to changes in the orbital combination of bonds. In this respect, the chemical activity of $\mathrm{s} / \mathrm{o}-X$ structures exhibits changes from $\mathrm{b}-X$.

In conclusion, we predicted another type of single-layer phase of group-V elements consisting of buckled square and octagon rings. They are dynamically and thermally stable even in their freestanding form. We found that these s/o structures of $\mathrm{P}, \mathrm{As}, \mathrm{Sb}$, and $\mathrm{Bi}$ are semiconductors.

\section{ACKNOWLEDGMENTS}

The computational resources were provided by TUBITAK ULAKBIM, High Performance and Grid Computing Center (TR-Grid e-Infrastructure). S.C. acknowledges financial support from the Academy of Sciences of Turkey (TÜBA). This research was supported by the Research Fund of the Adnan Menderes University under Project No. FEF-16016.
[1] K. S. Novoselov, A. K. Geim, S. V. Morozov, D. Jiang, Y. Zhang, S. V. Dubonos, I. V. Grigorieva, and A. A. Firsov, Science 306, 666 (2004).

[2] K. S. Novoselov, A. K. Geim, S. V. Morozov, D. Jiang, M. I. Katsnelson, I. V. Grigorieva, S. V. Dubonos, and A. A. Firsov, Nature (London) 438, 197 (2005).

[3] K. Novoselov, D. Jiang, F. Schedin, T. J. Booth, V. V. Khotkevich, S. V. Morozov, and A. K. Geim, Proc. Natl. Acad. Sci. U.S.A. 102, 10451 (2005).

[4] J. N. Coleman et al., Science 331, 568 (2011).

[5] C. Ataca, H. Şahin, and S. Ciraci, J. Phys. Chem. C 116, 8983 (2012).

[6] E. Durgun, S. Tongay, and S. Ciraci, Phys. Rev. B 72, 075420 (2005).

[7] S. Cahangirov, M. Topsakal, E. Aktürk, H. Şahin, and S. Ciraci, Phys. Rev. Lett. 102, 236804 (2009); S. Cahangirov, M. Topsakal, and S. Ciraci, Phys. Rev. B 81, 195120 (2010).

[8] P. Vogt, P. DePadova, C. Quaresima, J. Avila, E. Frantzeskakis, M. C. Asensio, A. Resta, B. Ealet, and G. LeLay, Phys. Rev. Lett. 108, 155501 (2012).

[9] M. E. Dávila and G. LeLay, Sci. Rep. 6, 20714 (2016).

[10] H. Şahin, S. Cahangirov, M. Topsakal, E. Bekaroglu, E. Aktürk, R. T. Senger, and S. Ciraci, Phys. Rev. B 80, 155453 (2009).
[11] T. Y. Lu, X. X. Liao, H. Q. Wang, and J. C Zheng, J. Mater. Chem. 22, 10062 (2012).

[12] S. Lin, S. Zhang, X. Li, W. Xu, X. Pi, X. Liu, F. Wang, H. Wu, and H. Chen, J. Phys. Chem. C 119, 19772 (2015).

[13] A. G. Gökçe and E. Aktürk, Appl. Surf. Sci. 332, 147 (2015).

[14] C. J. Tong, H. Zhang, Y-N. Zhang, H. Liu, and L. M. Liu, J. Mater. Chem. A 2, 17971 (2014).

[15] P. Tsipas et al., Appl. Phys. Lett. 103, 261605 (2013).

[16] Z. Al Balushi et al., Nat. Mater. 15, 1166 (2016).

[17] R. H. Baughman and H. Eckhardt, J. Chem. Phys. 87, 6687 (1987).

[18] A. Hirsch, Nat. Mater. 9, 868 (2010).

[19] F. Diederics, Nature (London) 369, 199 (1994).

[20] G. Li, Y. Li, H. Liu, Y. Guo, Y. Li, and D. Zhu, Chem. Commun. 46, 3256 (2010).

[21] V. O. Özçelik and S. Ciraci, J. Phys. Chem. C 117, 2175 (2013).

[22] B. G. Kim and H. J. Choi, Phys. Rev. B 86, 115435 (2012).

[23] V. N. Popov and P. Lambin, Phys. Rev. B 88, 075427 (2013).

[24] S. B. Ram, M. Aaditya, and S. K. Abhishek, Sci. Rep. 4, 7164 (2014).

[25] S. Zhang, S. Liu, S. Huang, B. Cai, M. Xie, L. Qu, Y. Zou, Z. $\mathrm{Hu}, \mathrm{X}$. Yu, and H. Zeng, Sci. China Mater. 58, 929 (2015).

[26] W. Li, M. Guo, G. Zhang, and Y. W. Zhang, Phys. Rev. B 89, 205402 (2014). 
[27] L. Li, Y. Yu, G. J. Ye, Q. Ge, X. Ou, H. Wu, D. Feng, X. H. Chen, and Y. Zhang, Nat. Nanotechnol. 9, 372 (2014).

[28] V. O. Özçelik, O. U. Aktürk, E. Durgun, and S. Ciraci, Phys. Rev B 92, 125420 (2015).

[29] Z. Zhu and D. Tománek, Phys. Rev. Lett. 112, 176802 (2014).

[30] J. Guan, Z. Zhu, and D. Tománek, ACS Nano 8, 12763 (2014).

[31] M. Wu, H. Fu, L. Zhou, K. Yao, and X. C. Zeng, Nano Lett. 15, 3557 (2015).

[32] C. Kamal and M. Ezawa, Phys. Rev. B 91, 085423 (2015).

[33] Z. Zhang, J. Xie, D. Yang, Y. Wang, M. Si, and D. Xue, Appl. Phys. Express 8, 055201 (2015).

[34] D. Kecik, E. Durgun, and S. Ciraci, Phys. Rev. B 94, 205409 (2016); 94, 205410 (2016).

[35] F. Ersan, E. Aktürk, and S. Ciraci, J. Phys. Chem. C 120, 14345 (2016).

[36] O. U. Aktürk, V. O. Özçelik, and S. Ciraci, Phys. Rev. B 91, 235446 (2015); O. Üzengi Aktürk, E. Aktürk, and S. Ciraci, ibid. 93, 035450 (2016).

[37] S. Zhang, Z. Yan, Y. Li, Z. Chen, and H. Zeng, Angew. Chem., Int. Ed. 54, 3112 (2015).

[38] S. Zhang, Z. Yan, Y. Li, Z. Chen, and H. Zeng, Angew. Chem. 127, 3155 (2015).

[39] G. Wang, R. Pandey, and S. P. Karna, ACS Appl. Mater. Interfaces 7, 11490 (2015).

[40] L. Cheng, H. Liu, X. Tan, J. Zhang, J. Wei, H. Lv, J. Shi, and X. Tang, J. Phys. Chem. C 118, 904 (2014).

[41] L. E. Díaz-Sánchez, A. H. Romero, M. Cardona, R. K. Kremer, and X. Gonze, Phys. Rev. Lett. 99, 165504 (2007).

[42] H. Iwasaki and T. Kikegawa, Acta Crystallogr., Sect. B 53, 353 (1997).

[43] E. Aktürk, O. U. Aktürk, and S. Ciraci, Phys. Rev. B 94, 014115 (2016).

[44] W. Yu, C. Y. Niu, Z. Zhu, X. Wang, and W. B. Zhang, J. Mater. Chem. C 4, 6581 (2016).

[45] L. Kou, X. Tan, Y. Ma, H. Tahini, L. Zhou, Z. Sun, A. Du, C. Chen, and S. C. Smith, 2D Mater. 2, 045010 (2015).

[46] S. Grimme, J. Comput. Chem. 27, 1787 (2006).

[47] P. E. Blöchl, Phys. Rev. B 50, 17953 (1994).

[48] J. P. Perdew, K. Burke, and M. Ernzerhof, Phys. Rev. Lett. 77, 3865 (1996).

[49] G. Kresse and J. Furthmuller, Phys. Rev. B 54, 11169 (1996).
[50] H. J. Monkhorst and J. D. Pack, Phys. Rev. B 13, 5188 (1976).

[51] A. Togo and I. Tanaka, Scr. Mater. 108, 1 (2015).

[52] J. Heyd, G. E. Scuseria, and M. Ernzerhof, J. Chem. Phys. 118, 8207 (2003).

[53] J. Heyd, G. E. Scuseria, and M. Ernzerhof, J. Chem. Phys. 124, 219906 (2006).

[54] J. Paier, M. Marsman, K. Hummer, G. Kresse, I. C. Gerber, and J. G. Ángyán, J. Chem. Phys. 124, 154709 (2006).

[55] J. Paier, M. Marsman, K. Hummer, G. Kresse, I. C. Gerber, and J. G. Ángyán, J. Chem. Phys. 125, 249901 (2006).

[56] M. Sun, S. Wang, J. Yu, and W. Tang, Appl. Surf. Sci. 392, 46 (2017).

[57] V. Vierimaa, A. V. Krasheninnikov, and H. P. Komsa, Nanoscale 8, 7949 (2016).

[58] Q. Wei and X. Peng, Appl. Phys. Lett. 104, 251915 (2014).

[59] J. W. Jiang and H. S. Park, J. Phys. D 47, 385304 (2014).

[60] J. Lee, W. C. Tian, W. L. Wang, and D. X. Yao, Sci. Rep. 5, 11512 (2015).

[61] B. Noläng, O. Eriksson, and B. Johansson, J. Phys. Chem. Solids 51, 1025 (1990).

[62] J. R. Feng and G. C. Wang, RSC Adv. 6, 22277 (2016).

[63] H. Kim, J. Korean Phys. Soc. 64, 547 (2014).

[64] Y. Aierken, D. Çakır, C. Sevik, and F. M. Peeters, Phys. Rev. B 92, 081408 (2015).

[65] J. Guan, Z. Zhu, and D. Tománek, Phys. Rev. Lett. 113, 046804 (2014).

[66] H. Guo, N. Lu, J. Dai, X. Wu, and X. C. Zeng, J. Phys. Chem. C 118, 14051 (2014).

[67] S. Mardanya, V. K. Thakur, S. Bhowmick, and A. Agarwal, Phys. Rev. B 94, 035423 (2016).

[68] M. Y. Liu, Y. Huang, Q. Y. Chen, C. Cao, and Y. He, Sci. Rep. 6, 29114 (2016).

[69] L. Kou, Y. Ma, X. Tan, T. Frauenheim, A. Du, and S. Smith, J. Phys. Chem. C 119, 6918 (2015).

[70] C. Kittel, Introduction to Solid State Physics, 8th ed. (Wiley, New York, 1996).

[71] C. S. Barrett, P. Cucka, and K. Haefner, Acta Crystallogr. 16, 451 (1963).

[72] M. Topsakal, S. Cahangirov, and S. Ciraci, Appl. Phys. Lett. 96, 091912 (2011).

[73] D. Singh, S. K. Gupta, Y. Sonvane, and I. Lukačević, J. Mater. Chem. C 4, 6386 (2016). 